\title{
Biomechanical comparison of distal locking screws for distal tibia fracture intramedullary nailing
}

\author{
Brennen L. Lucas ${ }^{1}$, Alexander C. M. Chong ${ }^{1,2}$, Bruce R. Buhr ${ }^{1}$, \\ Teresa L. Jones ${ }^{1}$, Paul H. Wooley ${ }^{1,2}$ \\ ${ }^{1}$ Department of Surgery, Section of Orthopaedics, The University of Kansas School of Medicine, Wichita, Kansas, USA; \\ ${ }^{2}$ Orthopaedic Research Institute, Wichita, Kansas, USA. \\ Email: alexander.chong@viachristi.org
}

Received 23 February 2011; revised 1 March 2011; accepted 3 March 2011.

\begin{abstract}
Background: Newer generation intramedullary (IM) tibial nails provide several distal interlocking screw options. The objectives were to determine: 1 ) if the new oblique interlocking option provides superior stability, 2) which screw orientation/configuration is the most biomechanically stable, and 3 ) if three distal interlocking screws provide better stability. Methods: A preliminary experiment was performed in torsion, compression, and bending tests with four different screw configurations: (I) one medial-tolateral and one oblique, (II) two medial-to-lateral, (III) one medial-to-lateral and one anterior-to-posterior, and (IV) one medial-to-lateral, one anterior-to-posterior and one oblique in simulated distal metaphyseal fracture tibiae. Twenty-four Synthes EXPERT tibial IM nails were used for six specimens of each screw configuration. Parts I and II, tibial IM nails were locked with $5.0 \mathrm{~mm}$ interlocking screws into simulated distal tibiae (PVC and composite analogue tibia). Part III, the two most stable configurations were tested using five pairs of simulated cadaveric distal tibiae metaphyseal fractures. Results: Significant differences were attributable to distal screw orientation for intramedullary nailing of distal tibia fractures. Configurations II and IV were found to be more stable than the other two configurations. No significant difference was detected in construct stability in all modes of testing between Configurations II and IV. Discussion: Configuration I did not provide superior stability for the distal tibia fracture fixation. Configurations II and IV provided equivalent stability. When choosing IM fixation for treatment of distal tibia metaphyseal fractures two medial-to-lateral screws provide the necessary stability for satisfactory fixation. Clinical Relevance: This study indi-
\end{abstract}

cated an option for operative treatment of distal metaphyseal tibia fracture fixation where preservation of soft tissue and rigid stabilization are needed.

Keywords: Biomechanical; Locking Screws; Intramedullary Nail; Distal Tibia

\section{INTRODUCTION}

The treatment of diaphyseal tibia fractures using intramedullary nailing is widely accepted and has been expanded to distal metaphyseal fractures [1-3]. Intramedullary nailing of diaphyseal tibia fractures offers a variety of advantages compared to other treatments including fracture stabilization with early weight bearing and good preservation of the soft tissue envelope [4-9]. One reason for treating distal fractures with intramedullary nailing is to protect the already damaged and violated thin soft tissue envelope [1-3,10-12]. Newer generation nail designs have been introduced that make prior nail modification techniques unnecessary and provide several distal interlocking screw options. For example, the Synthes EXPERT tibial nail system (Synthes USA, West Chester, PA) offers four different distal interlocking options. There are two medial to lateral screw options with the most proximal being $37 \mathrm{~mm}$ from the distal tip and the other medial to lateral screw hole located $13 \mathrm{~mm}$ from the tip of the nail. There is also an anterior to posterior option located $22 \mathrm{~mm}$ from the nail tip and a $30^{\circ}$ oblique (either direction) hole that is just $5 \mathrm{~mm}$ from the tip. In the technique guide "three locking screws" are advocated for distal fractures.

The literature conveys neither a consensus on the orientation of distal interlocking screws in distal metaphyseal tibia fractures, nor a consensus on the optimal number of distal interlocking screws. Two medial to lateral screws usually take less time to implant compared to a medial to lateral screw and an anterior to posterior 
screw, and it is much quicker to implant two screws compared with three screws. However, the question remains as to whether three distal interlocking screws confer greater fixation stability than two distal interlocking screws for distal fracture patterns.

Controversy has emerged surrounding the optimal number and configuration of interlocking screws for intramedullary nailing [3,13-19]. To our knowledge, in testing the detailed comparison of distal screw orientation and/or configuration for intramedullary nailing distal tibia fractures, little, if any, attempt has been made. The objectives of this study were: 1 ) to determine if the new distal oblique interlocking option provides superior stability, 2) to determine which screw orientation or configuration is the most biomechanically stable for the intramedullary nailing of distal tibia fractures, and 3) to determine if three distal interlocking screws provide any advantage in comparison to two distal interlocking screws. The null hypotheses for the present study were: 1) two distal interlocking screws, one medial to lateral and one oblique orientation, will provide equal construct stability when compared to the other interlocking screw configurations (two medial to lateral screws; a medial to lateral and an anterior to posterior screw; and a three screw configuration with a medial to lateral, an anterior to posterior and an oblique screw), and 2) two distal interlocking screws will provide as much fixation stability as three distal interlocking screws for distal metaphyseal fractures fixation.

\section{MATERIAL AND METHODS}

The study was conducted in three parts: The first two parts involved a preliminary experimental with four different screw configurations: (I) one medial to lateral and one oblique, (II) two medial to lateral, (III) one medial to lateral and one anterior to posterior, and (IV) one medial to lateral, one anterior to posterior and one oblique in simulated distal tibiae with a simulated distal metaphyseal fracture (Figure 1), while the third part compared the two most biomechanically stable configurations using a simulated distal cadaveric tibiae metaphyseal fracture. For all three parts, a total of twenty-four Synthes EXPERT tibial intramedullary nails $(10.0 \mathrm{~mm}$ diameter, $330 \mathrm{~mm}$ length, Synthes USA, West Chester, PA) (Figure 2) were used for this study.

For Part I of the biomechanical study, tibial intramedullary nails were distally locked with either two or three $5.0 \mathrm{~mm}$ interlocking screws into simulated distal tibiae consisting of PVC pipe (outer diameter $25.0 \mathrm{~mm}$ with inner diameter $17.0 \mathrm{~mm}$ ) (Figure 3(a)). The PVC pipe construct was chosen based on previous studies that showed PVC pipe, which served as a synthetic homogeneous material, has a comparable pullout and torque

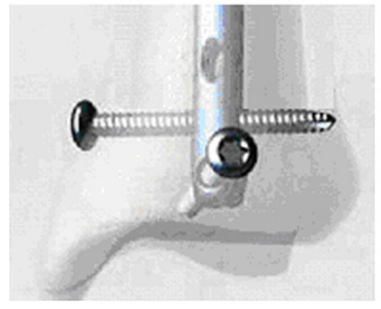

Configuration I

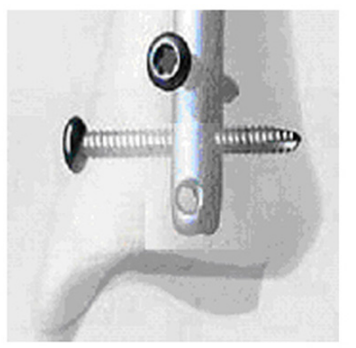

Configuration III

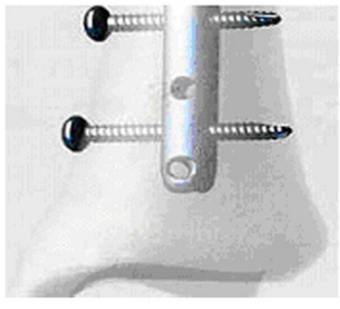

Configuration II

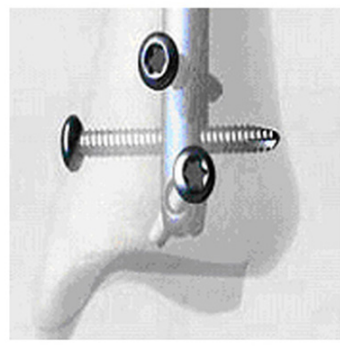

Configuration IV
Figure 1. Screw configurations.

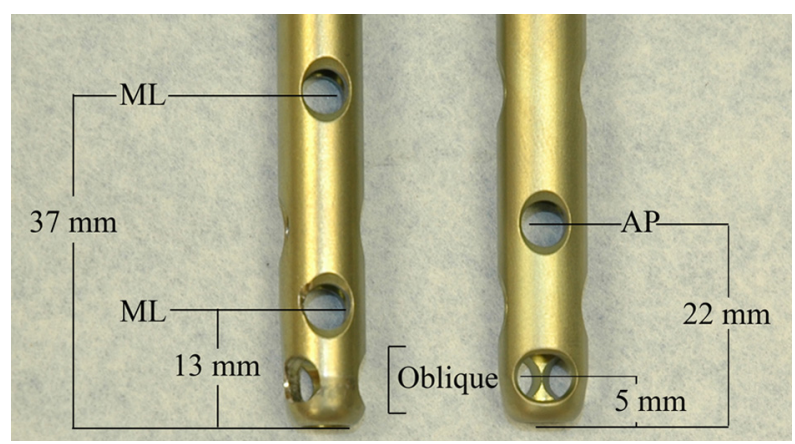

Figure 2. Synthes EXPERT tibial intramedullary nails.

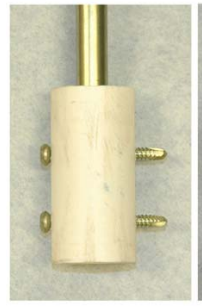

(a)

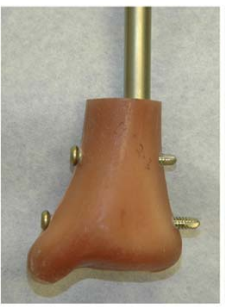

(b)

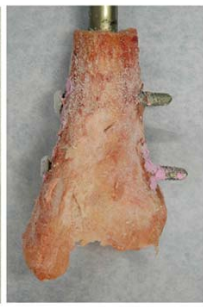

(c)

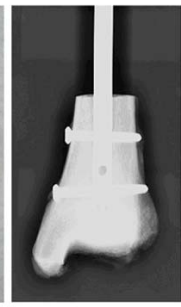

(d)
Figure 3. Samples of the experiment test specimens. (a) Part I test specimen (PVC), (b) Part II test specimen (composite analogue bone), (c) Part III test specimen (Cadaver), and (d) Radiograph of Part III test specimen.

strength for screws to those of bone [20,21]. To prepare the PVC pipe for implantations, each PVC pipe and the IM nail were placed in a custom designed holding jig to standardize the implantation location for those inter- 
locking screws were placed for each construct. The large simulated canal was used to represent a worst-case scenario with the distal tibia fracture with no endosteal to nail contact at the fracture site, and was designed to not allow the PVC pipe to limit deflection. Each specimen was then locked with another custom designed holding jig which centered with the nail, and fixed to the base of a MTS materials-testing apparatus (Figure 4(a) and Figure 5(a)). A total of twenty-four specimens were used for six specimens of each screw configuration.

For Part II, the test setup is similar as Part I, except the simulated distal tibiae consisting of composite distal tibia which represents simulated distal tibial metaphyseal fracture (Figure 3(b)). A total of twenty-four Four-Generation composite analogue distal tibiae (model \#3402, Pacific Research Laboratories, Inc., Vashon, WA) were used for six specimens of each screw configuration. To prepare the distal tibial metaphyseal fracture, each composite analogue distal tibia was placed in a custom designed cutting jig to generate a standardized transverse section made $40 \mathrm{~mm}$ from the tibiotalar surface. This fracture type was used to represent a very distal tibial fracture (OTA 43A) which eliminates any stability obtained from cortical contact between the proximal and distal fracture fragments. The distal tibial canal was reamed to $11 \mathrm{~mm}$ in diameter, then each composite analogue distal tibia and the IM nail were then placed in another custom designed holding jig to standardize the implantation location for those interlocking screws were placed for each construct. The results of Parts I and II were analyzed, and the two most stable configurations were determined and tested in Part III.

For Part III, the two most biomechanically stable screw configurations were applied in a cadaver biomechanical study with the distal tibia as the only source of bias (Figures 3(c) and 3(d)). Five pairs of cadaveric distal tibiae were used for each screw configuration tested. The bones were stripped of all soft tissue attachments and a simulated metaphyseal fracture was created by a transverse section made four centimeters from the tibiotalar surface, and the distal tibial canal was reamed to $11 \mathrm{~mm}$ in diameter, this procedure is similar to Part II. The fibula was detached from the tibia and was not included in the testing. The $10 \mathrm{~mm}$ Synthes EXPERT tibial intramedullary nails were then inserted to $5 \mathrm{~mm}$ above the distal tibial articular surface. Using fluoroscopy, the $5.0 \mathrm{~mm}$ distal interlocking screws were inserted in one of the two predetermined configurations using freehand technique. Each specimen was then potted in polymethylmethacrylate (PMMA) onto the mechanical testing jig centered within the nail, and the other end of the nail fixed to the base of a MTS materials-testing apparatus. The distal interlocking screws were protected with

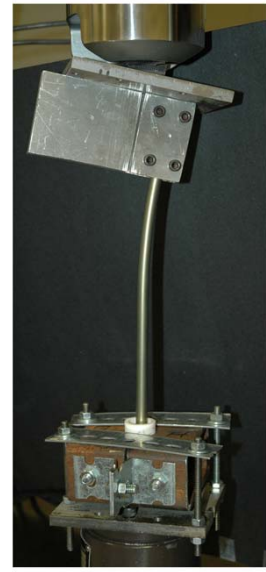

(a)

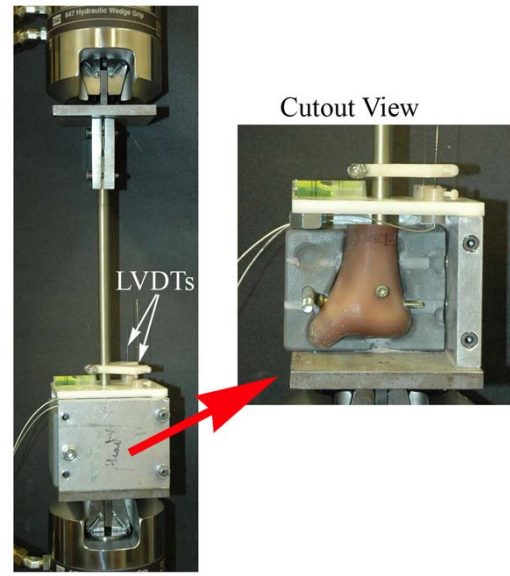

(b)
Figure 4. Compression and torsional experimental setup. (a) Part I experimental setup, (b) Parts II and III experimental setup.

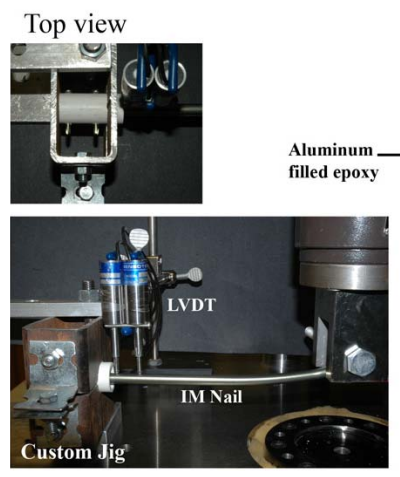

(a)
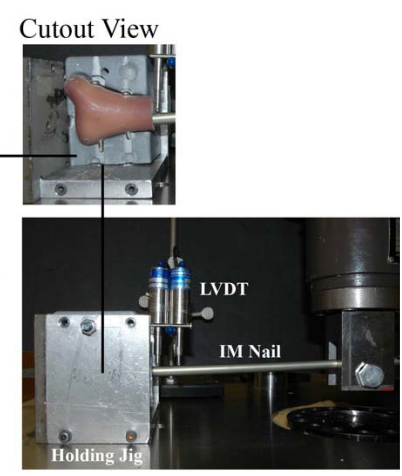

(b)
Figure 5. Bending experimental setup. (a) Part I experimental setup, (b) Parts II and III experimental setup.

soft molding compound (Play-Doh ${ }^{\circledR}$ brand) to prevent contact between the interlocking screw and the PMMA.

Each intramedullary nail/distal tibia model (Parts I, II and III) was tested under compressive loading, anterior-posterior bending, medial-lateral bending, and rotation torque in a Bionix servohydraulic materials testing system (MTS Model 858, Eden Prairie, MN). These tests were carried out within the elastic range of the constructs. For the deflection measurements of compressive loading, anterior-posterior bending, and medial-lateral bending, a completed two-dimensional motion across the fracture site in the loading (frontal) plane was measured with two linear variable differential transformers (LVDT) which determined displacements produced by the applied loads. These two LVDTs provided real time displacement measurements between the two points of attachment during testing, and the angular deflections were calculated using simple trigonometric principles. Only for the torque-angular displacement measurements 
were measured and collected from the MTS. For the load measurements of all test parameters were measured and collected by the MTS system for analysis. The average and standard deviation of the series were calculated for each type of construct in the corresponding test, and each specimen was tested in random order for all test parameters.

For the compression load tests (Figure 4), each specimen was loaded from $15 \mathrm{~N}$ in tension to a maximum load of $-1000 \mathrm{~N}$ (weight of a 225lb man) in compression at a rate of $10 \mathrm{~N} / \mathrm{sec}$. The $15 \mathrm{~N}$ in tension was according to Osterkamp [22] where he concluded that the proportions of the weight of a foot was $1.5 \%$ bodyweight, and the maximum load of $1000 \mathrm{~N}$ was chosen based on the procedure previously used by Gorczca et al [2]. Testing was initiated with two preconditioning loading cycles, and then the load-deflection data from the third to the fifth trials was recorded. Nail axial displacement measurements were taken at two points $90^{\circ}$ apart. This procedure was repeated three times for each specimen, removing and repositioning the specimen every time.

For the anterior-posterior and medial-lateral bend tests (Figure 5), the load was sinusoidal cycled from $\pm 10 \mathrm{~N}$ to $\pm 100 \mathrm{~N}$ for 5 cycles at a frequency of $0.05 \mathrm{~Hz}$. The corresponding bending moment varied from $\pm 2.5 \mathrm{Nm}$ to $\pm 25.0 \mathrm{Nm}$. Testing was initiated with two preconditioning loading cycles and then followed by three data collection loading cycles while force and displacement data were collected. This procedure was repeated three times for each specimen for each surface, removing and repositioning the specimen every time.

Rotation stability was tested at a frequency of $0.5 \mathrm{~Hz}$ from $-7.7 \mathrm{Nm}$ to $+7.7 \mathrm{Nm}$ of torque with $10 \mathrm{~N}$ of compression (Figure 4). The maximum torque applied (7.7 $\mathrm{Nm}$ ) in this investigation was approximately one-third of physiologic torques observed during normal activities [16]. Testing was initiated with two preconditioning torque cycles and then torque and rotation angle were recorded for the following three cycles. This procedure was repeated three times for each specimen while removing and repositioning the specimen each time.

\section{STATISTICAL ANALYSIS}

Data retrieved for Parts I and II from compression loading, anterior-posterior bending, medial-lateral bending, and rotation torque tests were analyzed with one-way analysis of variance (ANOVA) of SPSS software (Version 16.0; SPSS, Chicago, IL). Post hoc analysis of construct was used the Least Significant Difference (LSD) for multiple comparisons. The level of significant difference was defined as $\mathrm{p}<0.05$. These analyses were used to compare the difference in each screw configura- tion/orientation of distal locking screws in distal metaphyseal tibia fractures and each testing mode between constructs in term of stability. For Part III, paired t-tests were used to statistically evaluate the difference between the two most stable configurations, which determined from Parts I and II, for constructs stability. The level of significant difference was defined as $\mathrm{p}<0.05$.

\section{RESULTS}

For Part I, when compared in the compressive loading (range: $+15 \mathrm{~N}$ to $-1000 \mathrm{~N}$ ), anterior-posterior bending with low $( \pm 10 \mathrm{~N})$ and high $( \pm 100 \mathrm{~N})$ applied load, medial-lateral bending with low $( \pm 10 \mathrm{~N})$ and high $( \pm 100 \mathrm{~N})$ applied load, and rotation torque with low $( \pm 0.1 \mathrm{Nm})$ and high $( \pm 7.7 \mathrm{Nm})$ applied load, significant differences were detected in stability between all four screw configurations. Figure 6 shows the comparison of the construct stability properties between the four different screw configurations in Part I (PVC pipe) of the biomechanical study. For two screws configuration of all test modes, Configuration II was found to be more stable compared to the other two configurations. Configurations II and IV were found to be more stable than the other two configurations. Even though there were significant differences were detected between all four configurations, but from the post hoc analysis of construct there were only two significant differences were detected in stability in all modes of testing between two configurations: 1) Configurations I and II, and 2) Configurations II and III (Figure 6).

For Part II, significant differences were also detected in stability between all four screw configurations, and Figure 7 shows the construct stability properties for the four different screw configurations using composite analogue tibia. Configurations III and IV were found to be more stable than the other two configurations. Even though there were significant differences were detected between all four configurations, but from the post hoc analysis of construct there were only two significant differences were detected in stability in all modes of testing between two configurations: 1) Configurations I and IV, and 2) Configurations II and IV (Figure 7). Since there was no significant difference detected between Configurations II and III, and since Configuration II is the standard of care fixation configuration used currently, therefore, from Parts I and II results, Configurations II and IV were chosen to perform the cadaver biomechanical study.

For Part III, Figure 8 shows the construct stability properties for Configurations II and IV using cadaveric distal tibia. There was no significant difference detected in construct stability in all modes of testing between these two configurations. The one exception was mediallateral bending with low applied load. 


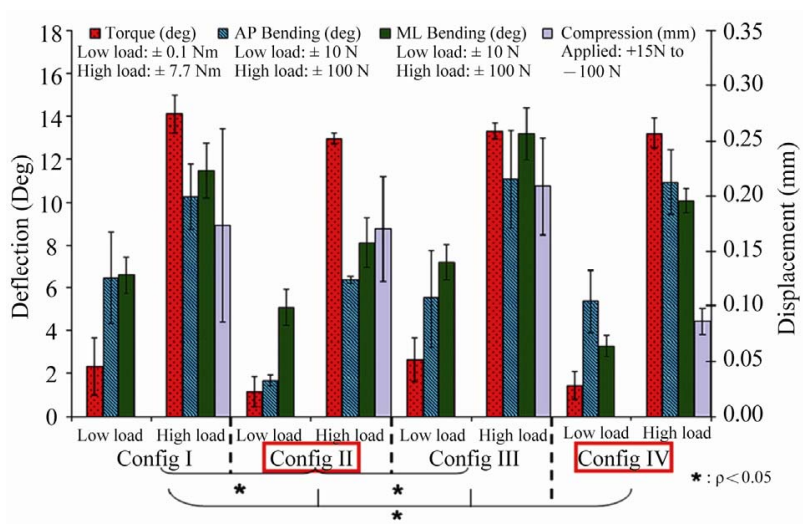

Figure 6. Construct stability properties for each screw configuration of Part I.

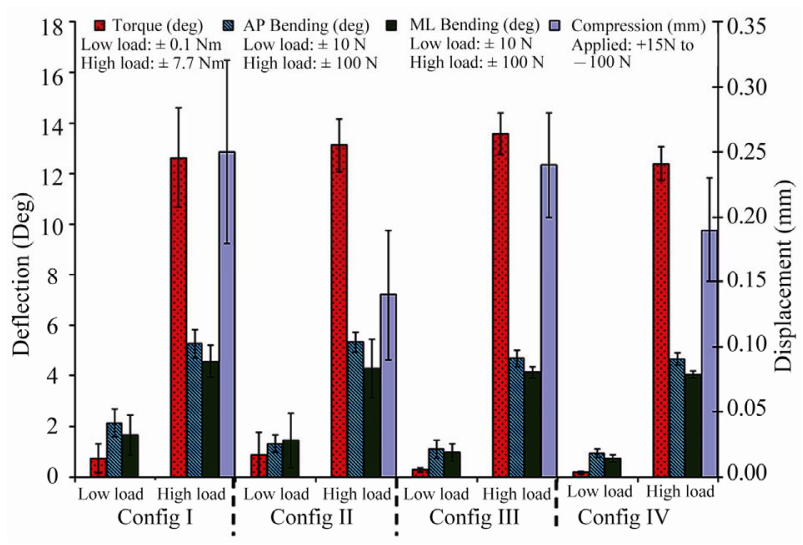

Figure 7. Construct stability properties for each screw configuration of Part II.

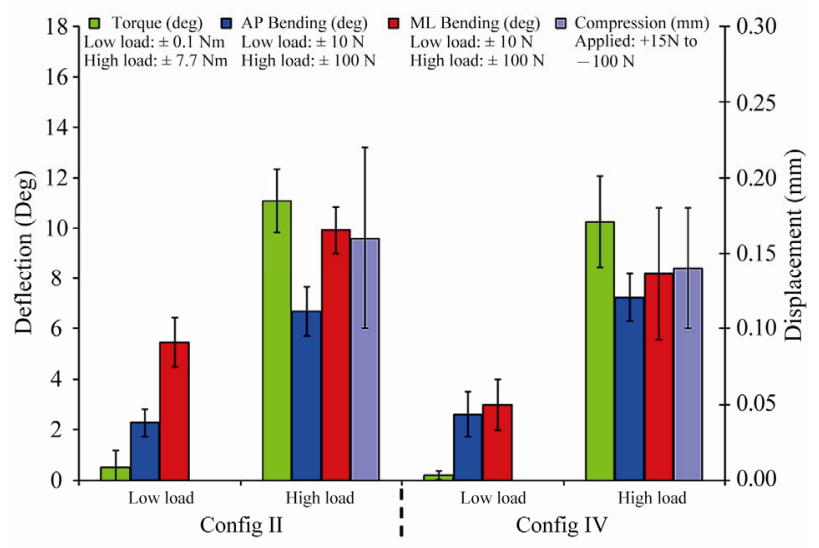

Figure 8. Construct Stability Properties Using Cadaveric Distal Tibia.

\section{DISCUSSION}

This study compared biomechanical Synthes EXPERT tibial nail stability using a variety of distal tibial locking screw configurations applied in intramedullary fixation. This tibial nail system has two distal oblique holes to enable the fixation of distal tibia fracture if necessary, and offers four different distal interlocking options. Our model assumed an unstable fracture pattern in the worst-case scenario of cortical bone loss or extensive comminution in which angular deflection would be limited only by distal interlocking screw configuration and position. The authors recognize that in some fracture patterns, cortical contact of the tibia or fibula would add to the stability of the bone-implant construct, however, this testing model was designed to examine the worst case scenario.

Kneifel and Buckley [17] demonstrated an increased rate of failure using one distal interlocking screw compared to two distal interlocking screws with unreamed nails in tibial shaft fractures. Chen et al. [15] showed that there is no statistical difference in nail stability between two parallel (medial to lateral) and two perpendicular (one medial to lateral, one anterior to posterior) nails in anterior, posterior, medial or lateral directions, or torsional loading. However, the results of this study indicated significant differences in construct stability between all four screw configurations for distal tibia fracture fixation using intramedullary nailing. This study also investigated whether the new distal oblique interlocking option (Configuration I) added to the stability of the fixation of the distal tibia fracture, but the results showed that this oblique interlocking option did not provide superior stability for the fixation of the distal tibia fracture.

The current biomechanical study (Part I and Part II) using PVC pipe and composite analogue tibia as simulated distal tibiae indicated that Configuration II and Configuration IV were more biomechanically stable for the intramedullary nailing of distal tibia fractures when compared to the other two configurations. Our results are in agreement with Chen et al. [15], and concur that the instability of the screw fixation is probably due to screw movement within the interlocking screw-hole, and the mismatch between the screw threads and the interlocking screw-hole. Chen et al. [15] also determined that there were possible two types of screw movements: Tilt and shift. Our current study using the Synthes EXPERT tibial nail system measured the worse possible tilt and torsional movement using the dimension of the mismatch between the screw threads and the interlocking screw-hole, which was found to be approximately $2.9^{\circ}$ (range: $2.7^{\circ}-3.1^{\circ}$ ) (Figure 9).

A relatively small number of cadaveric samples were included in this study; however, the changes in the construct stability properties between two distal interlocking screws (Configuration II) and three distal interlocking 

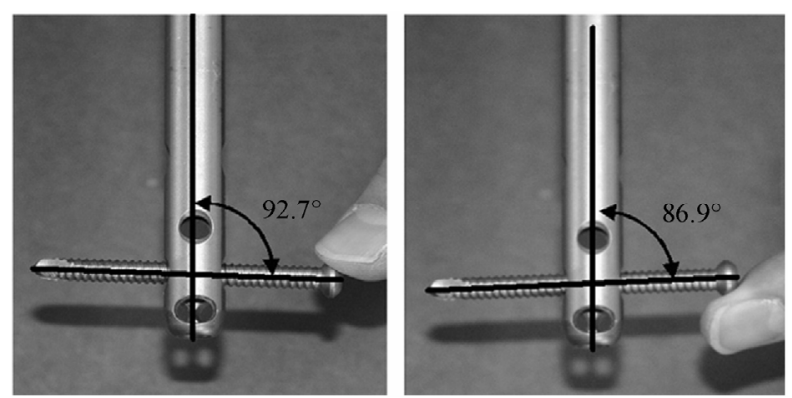

Figure 9. Screw movement within the interlocking screw-hole.

screws (Configuration IV) were extremely small and repeatable. Therefore, we are confident that two medial to lateral distal interlocking screws (Configuration II) and three distal interlocking screws (Configuration IV) provide equivalent construct stability.

When the results from the three simulated distal tibia models with a simulated distal metaphyseal fracture were compared (Figures 6-8), significant differences (p $<0.05)$ were detected. This was especially true for ML bending under high load, where the deflection with cadaveric model was more than twice than that with composite analogue bone model. In this study, only one size of the tibial intramedullary nail (10.0 $\mathrm{mm}$ diameter) was used, and different bone sizes occurring within the cadaveric specimens might be a limiting factor in this investigation. Therefore, the cadaveric findings might reflect a higher variable than the composite analogue bone model within the comparison. In fact, when the comparison is performed within the same simulated test model, the magnitude of the difference in all modes is much smaller. Further clinical research is needed to confirm our results.

The information currently available in the literature conveys neither a consensus on the orientation of distal interlocking screws in distal metaphyseal tibia fractures, nor agreement on the optimal number of distal interlocking screws. The reason could be because either cadaveric specimens or patient bones have significant variations in size and bone quality and require the use of different intramedullary nails sizes. Therefore a valid conclusion cannot be construed. In this laboratory investigation using the composite analogue tibiae, low inter sample variability, and construct stability for several screw orientations allows direct comparison between each configuration. However, the results of this laboratory studies cannot readily be extrapolated to clinical situation. A larger study, with higher number of cadaveric bones and with varying bone density, may demonstrate more clearly the screw configuration effect on the stability for the intramedullary nailing of distal tibia fractures.

There are some limitations to our study. Despite our efforts to insert all screws in the same perfect central screw placement, it is possible that drilling and inserting the screw may result in a small degree of tilt that might cause the nail and screw to interlock, in turn cause nail stability. However, we believe that screw insertion was performed as clinically intended (essentially by eye and fluoroscope). In addition, it may be more difficult to insert the oblique screw compared to the medial to lateral or anterior to posterior, despite our best efforts to standardize screw insertion. Drilling and redirection of the screws was avoided in this study. Another inherent limitation is that the inter-hole distance varied depending on the interlocking configuration used. Strict geometric considerations suggest that this favors Configurations II and IV, as the greater working length further restricts angulations. However, this design parameter is of practical importance and should be considered when deciding on distal interlocking screw orientation. George et al. [18] have shown that the further the distal interlocking screw is located from the fracture, the greater the rotation stability of the construct.

Overall, when choosing intramedullary fixation for the treatment of distal tibia metaphyseal fractures, our data suggest that two medial to lateral screws may provide the necessary biomechanical stability for satisfactory fixation and clinical beneficial, because this not only saves operative and fluoroscopy time, but also may be economically advantageous compared to three distal locking screws and/or an oblique screw.

\section{CONFLICT OF INTEREST STATEMENT}

This study received a support grant from Synthes (West Chester, PA, USA) with approved the study design, and provide the Synthes EXPERT tibial nail system used in this study. Pacific Research Laboratories, Inc. (Vashon Island, WA) did provide the analogue tibia. Both Pacific Research Laboratories, Inc. and Synthes USA had no role in the collection, analysis and interpretation of data, in the writing of the manuscript, or in the decision to submit the manuscript for publication. This study also did not receive any payments or other personal benefit or a commitment or agreements that were related in any way to the subject of the research that we conducted in connection with the research.

\section{ACKNOWLEDGEMENTS}

The authors thank Synthes USA (West Chester, PA) for providing the Synthes EXPERT tibial nail system used in this study. The authors also wish to thank Mr. Joel D. White and Mr. Preston T. Saal for their assistant on data entering on this study. No benefits of any form have been received directly or indirectly to the subject of this article, and 
there is no any potential of conflict of interest.

\section{REFERENCES}

[1] Dogra, A.S., Ruiz, A.L., Thompson, N.S. and Nolan, P.C. (2000) Dia-metaphyseal distal tibial fractures-treatment with a shortened intramedullary nail: A review of 15 cases. Injury, 31, 799-804.

[2] Gorczyca, J.T., McKale, J., Pugh, K. and Pienkowski, D. (2002) Modified tibial nails for treating distal tibia fractures. Journal of Orthopaedic Trauma, 16, 18-22. doi:10.1097/00005131-200201000-00004

[3] Fan, C.Y., Chiang, C.C., Chuang, T.Y., Chiu, F.Y. and Chen, T.H. (2005) Interlocking nails for displaced metaphyseal fractures of the distal tibia. Injury, 36, 669-674. doi:10.1016/j.injury.2004.10.018

[4] Alho, A., Ekeland, A., Stromsoe, K., Folleras, G. and Thoresen, B.O. (1990) Locked intramedullary nailing for displaced tibial shaft fractures. The Journal of Bone and Joint Surgery, British Volume, 72, 805-809.

[5] Blachut, P.A., O’Brien, P.J., Meek, R.N. and Broekhuyse, H.M. (1997) Interlocking intramedullary nailing with and without reaming for the treatment of closed fractures of the tibial shaft. A prospective, randomized study. The Journal of Bone and Joint Surgery. American Volume, 79, 640-646.

[6] Bone, L.B. and Johnson, K.D. (1986) Treatment of tibial fractures by reaming and intramedullary nailing. The Journal of Bone and Joint Surgery. American Volume, 68, 877-887.

[7] Court-Brown, C.M., Christie, J. and McQueen, M.M. (1990) Closed intramedullary tibial nailing. Its use in closed and type I open fractures. The Journal of Bone and Joint Surgery. British Volume, 72, 605-611.

[8] Dehne, E., Metz, C.W., Deffer, P.A. and Hall, R.M. (1961) Nonoperative treatment of the fractured tibia by immediate weight bearing. Journal of Trauma, 1, 514-535.

[9] Sarmiento, A., Gersten, L.M., Sobol, P.A., Shankwiler, J.A. and Vangsness, C.T. (1989) Tibial shaft fractures treated with functional braces. Experience with 780 fractures. The Journal of bone and joint surgery. British volume, 71, 602-609.

[10] Im, G.I. and Tae, S.K. (2005) Distal metaphyseal fractures of tibia: A prospective randomized trial of closed reduction and intramedullary nail versus open reduction and plate and screws fixation. Journal of Trauma, 59, 1219-1223 (Discussion 1223). doi:10.1097/01.ta.0000188936.79798.4e

[11] Nork, S.E., Schwartz, A.K., Agel, J., Holt, S.K., Schrick, J.L. and Winquist, R.A. (2005) Intramedullary nailing of distal metaphyseal tibial fractures. The Journal of Bone and Joint Surgery. American Volume, 87, 1213-1221. doi:10.2106/JBJS.C.01135
[12] Andrew, H.S., Christopher, G.F. and Paul, T. III (2003) Treatment of closed tibial fractures. The Journal of Bone and Joint Surgery. American Volume, 85, 352-368.

[13] Mosheiff, R., Safran, O., Segal, D. and Liebergall, M. (1999) The unreamed tibial nail in the treatment of distal metaphyseal fractures. Injury, 30, 83-90. doi:10.1016/S0020-1383(98)00213-7

[14] Laflamme, G.Y., Heimlich, D., Stephen, D., Kreder, H.J. and Whyne, C.M. (2003) Proximal tibial fracture stability with intramedullary nail fixation using oblique interlocking screws. Journal of Orthopaedic Trauma, 17, 496502. doi:10.1097/00005131-200308000-00004

[15] Chen, A.L., Tejwani, N.C., Joseph, T.N., Kummer, F.J. and Koval, K.J. (2001-2002) The effect of distal screw orientation on the intrinsic stability of a tibial intramedullary nail. Bulletin (Hospital for Joint Diseases (New York, N.Y.)), 60, 80-83.

[16] Roberts, C.S., King, D., Wang, M., Seligson, D. and Voor, M.J. (1999) Should distal interlocking of tibial nails be performed from a medial or a lateral direction? anatomical and biomechanical considerations. Journal of Orthopaedic Trauma, 13, 27-32. doi:10.1097/00005131-199901000-00007

[17] Kneifel, T. and Buckley, R. (1996) A comparison of one versus two distal locking screws in tibial fractures treated with unreamed tibial nails: A prospective randomized clinical trial. Injury, 27, 271-273. doi:10.1016/0020-1383(95)00116-6

[18] George, C.J., Lindsey, R.W., Noble, P.C., Alexander, J.W. and Kamaric, E. (1998) Optimal location of a single distal interlocking screw in intramedullary nailing of distal third femoral shaft fractures. Journal of Orthopaedic Trauma, 12, 267-272. doi:10.1097/00005131-199805000-00009

[19] Gollwitzer, H., Karampour, K., Hauschild, M., Diehl, P., Busch, R. and Mittelmeier, W. (2004) Biomechanical investigation of the primary stability of intramedullary compression nails in the proximal tibia: Experimental study using interlocking screws in cryopreserved human tibias. Journal of Orthopaedic Science, 9, 22-28.

[20] Heidemann, W., Gerlach, K.L., Gröbel, K.H. and Köllner, H.G. (1998) Influence of different pilot hole sizes on torque measurements and pullout analysis of osteosynthesis screws. Journal of Cranio-Maxillo-Facial Surgery, 26, 50-55. doi:10.1016/S1010-5182(98)80035-8

[21] Heidemann, W., Gerlach, K.L., Gröbel, K.H. and Köllner, H.G. (1998) Drill Free Screws: A new form of osteosynthesis screw. Journal of Cranio-Maxillo-Facial Surgery, 26, 163-168. doi:10.1016/S1010-5182(98)80007-3

[22] Osterkamp, L.K. (1995) Current perspective on assessment of human body proportions of relevance to amputees. Journal of the American Dietetic Association, 95, 215-218. doi:10.1016/S0002-8223(95)00050-X 